\title{
Bioactive Fatty Acids Reduce Development of Gastric Cancer Following Duodenogastric Reflux in Rats
}

\author{
Bjørn Jostein Christensen ${ }^{1,2}$, Kjetil Berge ${ }^{3}$, Hege Wergedahl ${ }^{4}$, Pavol Bohov ${ }^{3}$, \\ Rolf Kristian Berge $^{3,5}$, Einar Svendsen ${ }^{6,7}$, Asgaut Viste ${ }^{1,2}$ \\ ${ }^{1}$ Department of Surgical Sciences, University of Bergen, Bergen, Norway \\ ${ }^{2}$ Department of Gastrointestinal and Acute Surgery, Haukeland University Hospital, Bergen, Norway \\ ${ }^{3}$ Institute of Medicine, University of Bergen, Bergen, Norway \\ ${ }^{4}$ Faculty of Education, Section for Food and Health, Bergen University College, Bergen, Norway \\ ${ }^{5}$ Department of Heart Disease, Haukeland University Hospital, Bergen, Norway \\ ${ }^{6}$ The Gade Institute, Section of Pathology, University of Bergen, Bergen, Norway \\ ${ }^{7}$ Department of Pathology, Haukeland University Hospital, Bergen, Norway \\ Email: bjch@helse-bergen.no
}

Received November 2, 2011; revised November 29, 2011; accepted December 16, 2011

\begin{abstract}
Background: Bioactive fatty acids such as the eicosapentaenoic acid (EPA) and docosahexaenoic acid (DHA) and the modified fatty acid analogue, tetradecylthioacetic acid (TTA), are known to influence inflammatory processes in the body. Our aim was to investigate if diets containing fish oil (FO) enriched with bioactive fatty acids could affect inflammation and development of glandular stomach carcinogenesis in a duodenogastric reflux (DGR) animal model. We also wanted to evaluate if a high-fat diet might increase the risk of developing gastric cancer compared to a low-fat diet. Methods: 185 rats operated on with a gastroenterostomy were randomly allocated to 5 different treatment groups given: low-fat, high-fat, high-fat + FO, high-fat + TTA or high-fat + FO + TTA. The stomachs were removed after 50 weeks and examined by light microscopy with hematoxylin and eosin staining (HE). Immunohistochemical staining against COX-2, PCNA and p53 was performed when adenocarcinomas were found. The plasma fatty acid profile was determined. Results: Adenocarcinomas developed in $21 \%$ of animals fed the low-fat diet, $35 \%$ in the high-fat group, $16 \%$ in the high-fat + TTA group, $21 \%$ in the high-fat + FO group and $8.6 \%$ in the high-fat + FO + TTA treatment group. COX-2 and PCNA were positive whereas p53 was negative in the majority of the samples. The anti-inflammatory fatty acid index increased after treatment with FO and in combination with FO and TTA. Conclusion: FO and TTA in combination with a high-fat diet significantly lower the risk of developing adenocarcinomas in rats subjected to duodenogastric reflux. This is most likely due to a selective modulation of inflammation.
\end{abstract}

Keywords: Bioactive Fatty Acids; Cancer; Duodenogastric Reflux; Experimental; Fish Oil; Gastric Carcinogenesis; Glandular Stomach; Immunohistochemistry; Rats; TTA

\section{Introduction}

Although the incidence of gastric cancer is declining in the Western countries, it is still among the most frequently occurring gastrointestinal (GI) cancers world-wide. It ranges as number two regarding cancer mortality [1,2]. Worldwide, the incidence of gastric cancer in the distal part of the stomach is decreasing, whereas the proportion of cancers in the proximal part of the stomach is increaseing. The reason for this might be a reduced incidence of Helicobacter pylori infections. Also, a general increase in body mass index and an increased occurrence of duodeno-gastric and gastroesophageal reflux in the western population may be correlated to the increased incidence of proximal cancers [3-5]. Some researchers have suggested that a diet with a high-fat content rich in saturated fatty acids (SFA) might explain the increase in GI cancers [6-12]. In order to influence this type of cancer development, it is relevant to modulate or even reduce the sequence of "chronic inflammation-metaplasia-dysplasia", which can lead to cancer development. Several experimental studies have shown that reduction of chronic inflammation with cyclooxygenase-2 (COX-2)-inhibitors can reduce the frequency of adenocarcinoma (AC) in the stomach and distal esophagus $[13,14]$. Based on epidemiologic studies, it has been suggested that a diet high in meat may increase the risk of distal stomach adenocarcinoma [15]. On the other hand, since the 1930's, it has been known that fish oil (FO) can have suppressive effects on tumor growth [16]. There is also epidemiological evi- 
dence that dietary omega-3 polyunsaturated fatty acids (PUFA) have beneficial effects against several malignnancies [17-19]. The mechanisms behind the anti-tumor activity of omega-3 PUFA are not fully understood, but there is some evidence that these fatty acids can promote apoptosis in cancer cells [20-22]. To enhance the efficiency of fatty acids to impair proliferation and expect pro-apoptotic effects, various attempts have been made to modify their molecular structure. One such fatty acid analogue, tetradecylthioacetic acid (TTA), is reported to reduce the proliferation of breast cancer, glioma, lymphomas and leukaemia cell lines. It also inhibits glioma xenograft transplants from growing, inhibits leukaemia cell growth in rats, and inhibits proliferation in acute myeloid leukaemia (AML) cell cultures [23-26]. The antiproliferative effects of TTA may be due to induction of apoptosis, possibly mediated by mitochondrial alterations and changes in inflammation signalling [27]. The aims of this study was to evaluate: 1 ) if a diet rich in saturated fat (25\%) would increase the risk of developing gastric cancer, as compared to a low-fat diet (7\%), and 2) if different types of bioactive fatty acids added to the high-fat diet could modulate gastric carcinogenesis.

\section{Methods}

\subsection{Animals}

Two hundred ten male Wistar rats, 8 weeks old on arrival, weight $267.6 \pm 29.8 \mathrm{~g}$ (Median \pm SD) were supplied by Taconic Europe A. Five rats were housed in each cage on aspen bedding at standard laboratory conditions with room temperature $22^{\circ} \mathrm{C} \pm 2^{\circ} \mathrm{C}$ and $55 \% \pm 10 \%$ relative humidity, at $12 \mathrm{~h} \mathrm{light/dark} \mathrm{cycle.} \mathrm{The} \mathrm{room} \mathrm{air} \mathrm{was} \mathrm{ex-}$ changed 18 times/h. The animals arrived at the facility at least 7 days before the experiments started and were handled according to the FELASA accreditation scheme (Federation of European Laboratory Animals Science Association, Utrecht, Netherlands) after arrival. The rats had continuously free access to tap water, and were fed commercial chow until start of the experiment. The study was approved by the experimental animal board of the Norwegian Department of Agriculture.

\subsection{Animal Preparation and Surgical Procedures}

All animals received only tap water $24 \mathrm{~h}$ prior to surgery. Before surgery the rats received buprenorphinum $(0.24$ $\mathrm{mg} / \mathrm{kg}$ ) subcutaneously. Anesthesia was induced and continued with spontaneously inhalation of $5 \%$ isofluran combined with a 50:50\% blend of nitrous oxide and oxygen circulating in a closed chamber for small animals. The abdomen was opened via an upper midline incision. The first jejunal loop was identified, and a one cm long side-to-side gastrojejunostomy was performed between this loop and the greater curvature of the glandular stomach immediately distal to the junction of the forestomach. The anastomosis was performed as an all layer continuous suture with 6 - 0 polyglactin. The abdominal wall was closed with continuous 4 - 0 silk suture and staples in the skin. After surgery the rats were kept in an incubator at $29^{\circ} \mathrm{C}$ until they regained mobility. They were allowed carbohydrates dissolved in tapwater immediately after surgery, and commercial chow after $48 \mathrm{~h}$. Buprenorphin $(0.12 \mathrm{mg} / \mathrm{kg})$ was given subcutaneously every 12 $\mathrm{h}$ for $36 \mathrm{~h}$, later by signs of discomfort.

\subsection{Study Design and Food Administration}

The one hundred and eighty-five rats that were included in this study, were chipped for identification, and randomly divided into five different treatment groups. Five animals were kept in each cage and had free access to water and diet. The 5 treatment groups included in the study were: 1) low-fat diet (LF) with 7\% saturated fat; 2) high-fat diet (HF) with 25\% saturated fat (23\% lard, $2 \%$ soybean oil); 3) HF diet supplemented with TTA (0.375\%); 4) HF diet supplemented with FO (10.4\% FO, $12.6 \%$ lard, $2 \%$ soy oil); and 5) HF diet supplemented with TTA (0.375\%) and FO (10.4\%). Complete diet compositions are given in Table 1. Casein was obtained from Tine BA, Oslo, Norway. TTA was provided by the Lipid Research Laboratory, Section of Medical Biochemistry, Institute of Medicine, Haukeland University Hospital, Norway. EPAX 6000 TG fish oil was kindly provided by EPAX AS, Ålesund, Norway. Lard was purchased from TenKate, Musselkanaal, The Netherlands and soya oil was commercially available. All other ingredients were obtained from Dyets Inc., Bethlehem, PA, USA. The pellets for each diet group were made by Nofima Ingrediens, Norway. The pellets were stored at $-20^{\circ} \mathrm{C}$ and thawed up before administration. At week 50, the rats were sacrificed by exsanguination using the same anesthesia as during surgery.

\subsection{Tissue Preparation}

After sacrificing the animal, the peritoneal cavity was explored for metastases. The stomach was removed along with the lower esophagus, duodenum and the small bowel anally of the anastomosis. The stomach was opened along the greater curvature, including the anastomosis, in a standardized way and pinned on a corkboard. The pinned specimen was fixed in $10 \%$ buffered formaldehyde for $24 \mathrm{~h}$ and then in $70 \%$ ethanol before a whole wall section was made from the forestomach, the glandular part with the anastomosis and the pyloric area. Strips of 3 - $4 \mathrm{~mm}$ thickness were cut stepwise from the anastomosis. The strips were dehydrated by increasing concentrations of 
Table 1. Composition of diets.

\begin{tabular}{lccccc}
\hline & Low-fat (LF) & High-fat (HF) & HF + TTA & HF + FO & HF + FO + TTA \\
\hline Casein & 156.0 & 196.9 & 196.9 & 196.9 & 196.9 \\
Lard & 50.0 & 230.0 & 226.3 & 126.0 & 122.3 \\
Soy bean oil & 20 & 20 & 20 & 20 & 20 \\
Fish Oil & 0 & 0 & 0 & 104 & 104 \\
TTA & 0 & 0 & 3.8 & 0 & 3.8 \\
Cornstarch & 572.2 & 351.3 & 351.3 & 351.3 & 351.3 \\
Sucrose & 100 & 100 & 100 & 100 & 100 \\
Fibre & 50 & 50 & 50 & 50 & 50 \\
AIN-93G-MX mineral mix & 35 & 35 & 35 & 35 & 35 \\
KH2PO4, monobasic & 1.3 & 1.3 & 1.3 & 1.3 & 1.3 \\
AIN-93-VX vitamin mix & 10 & 10 & 10 & 10 & 10 \\
L-Cystine & 3 & 3 & 3 & 3 & 3 \\
Choline bitartrate & 2.5 & 2.5 & 2.5 & 2.5 & 2.5 \\
tert-Butyl-hydroquinone & 0.014 & 0.014 & 0.014 & 0.014 & 0.014 \\
\hline
\end{tabular}

All numbers are given as $\mathrm{g} / \mathrm{kg}$ diet. Casein contained $83.8 \%$ protein and $0.2 \%$ water.

ethanol, orientated and embedded in paraffin wax. Sections of $4 \mu \mathrm{m}$ thickness were stained with hematoxylin and eosin (H \& E). Specimens where adenocarcinomas (AC) had developed were further studied with immunohistochemistry. As controls, five samples without adenocarcinoma development were randomly selected from each diet group. These were also studied with immunohistochemistry.

\subsection{Histopathological Analysis}

The fore-stomach with esophagus, the glandular stomach with the anastomosis and the pyloric region were searched for changes in gross anatomy and histopathology. Except for in the area of the anastomosis, no pathology nor peritoneal metastases were seen. According to classification used in similar reflux models, the changes were categorized as follows [28]:

1) Ulceration.

2) Cystic dilatation.

3) Adenocystic proliferation.

4) Adenocarcinoma.

The microscopy of the slides was done blindly by a specialist in gastrointestinal pathology.

\subsection{Immunohistochemical Analysis}

All sections positive for AC were stained for immunohistology with P53 (1:300, rabbit polyclonal; Thermo Scientific, UK), PCNA (1:1000, rabbit polyclonal; Thermo Scientific, UK) and COX-2 (1:200, rabbit poly- clonal; Thermo Scientific, UK) antibodies. After immune reaction, sections were treated with Dako EnVision (DakoCytomation, Denmark) and DAB (3,3'-diaminobenzidine tetrahydrochloride) was used to visualize the antigen-antibody reaction. Sections were counterstained with eosin to visualize the tissue. In addition, we stained for COX-2, PCNA and p53 in the gastric mucosa in samples where no AC was observed. For this purpose, 5 rats without AC and with normal microscopic gastric mucosa were picked randomly from each treatment group, in total 25 rats.

\subsection{Fatty Acid Analysis}

Total plasma lipids were extracted with chloroform: methanol (1:2) [29] and fatty acid methyl esters (FAME) were obtained by heating of lipids with methanol at $90^{\circ} \mathrm{C}$ for $1 \mathrm{~h}$, where $\mathrm{H}_{2} \mathrm{SO}_{4}$ was used as a catalyst [30]. After extraction into an organic solvent, FAMEs were analyzed by gas-liquid chromatography. Gas chromatograph GC 8000 TOP (Finnigan, USA) was equipped with a programmed temperature vaporization injector, flame-ionization detector, AS 800 auto sampler and with a fused silica capillary column coated with dimethylpolysiloxane stationary phase, DB1-ms (J \& W Scientific, USA). Hydrogen was used as carrier gas. Column temperature was programmed from $110^{\circ} \mathrm{C}$ to $310^{\circ} \mathrm{C}$ with a gradient $2.5^{\circ} \mathrm{C} /$ min. GC signal was acquired and evaluated with Chromeleon software (Dionex, USA). Peaks were identified by use of known fatty acid (FA) standards and by mass spectra obtained by GC/MS analysis (GCQ, Finnigan, 
USA) on the same column. Internal standard C21:0 was used for quantifying after calibration with known mixtures of FA standards. The anti-inflammatory fatty acid Index (AIFAI) was calculated as (docosapentaenoic acid + docosahexaenoic acid + dihomo- $\gamma$-linolenic acid + eicosapentaenoic acid) $\times$ 100/arachidonic acid. Slightly different indexes have been used by Chavali et al. and Utsunomiya et al. [31,32].

\subsection{Statistical Analysis}

Differences between the groups were analyzed by cross tabulation and two-sided Fisher's exact test. P-values < 0.05 were considered significant. One way ANOVA calculation was used for evaluation of the plasma lipid distribution between dietary groups. Statistics were performed by using PASW Statistics for Windows, version 16, (SPSS Inc., Chicago, IL, USA).

\section{Results}

12 rats died hours after the operation and 13 rats died within a few days postoperatively probably due to severe gastroesophageal reflux. The remaining 185 animals were included in the study and sacrificed after 50 weeks of treatment. The diets were well tolerated and had no apparent adverse effect on the rats.

\subsection{Macroscopic and Histopatological Findings}

Macroscopic examination of the stomachs revealed 60 tumors close to the gastrointestinal anastomosis (Figure 1). No tumors were localized in other areas of the stomach nor were metastases found in the peritoneal cavity. Histological evaluation by light microscopy did not confirm AC in all macroscopic tumors. The observed non-AC tumors were classified by histology as ulceration, adenocystic proliferation or cystic dilatation (Table 2). Of the 60 observed tumors, 38 were classified as AC. Figure 2 shows a typically AC at $100 \times$ magnification. The distribution of $\mathrm{AC}$ in the various treatment groups are depicted in Figure 3. Although not statistically signifycant, the occurrence of AC tended to be higher in the animals fed a HF diet (35\%), compared to animals fed a LF diet (21\%). Both TTA (16\%) and FO (21\%) tended to reduce the incidence of AC compared to animals fed HF diet alone. When comparing the $\mathrm{HF}+\mathrm{FO}+\mathrm{TTA}$ diet group with the HF diet group, we observed a statistically significant decrease in cancer frequency (8.6\%) compared to the HF group (35\%) ( $<<0.01$ ). By ANOVA testing we found no significant difference when treatments with

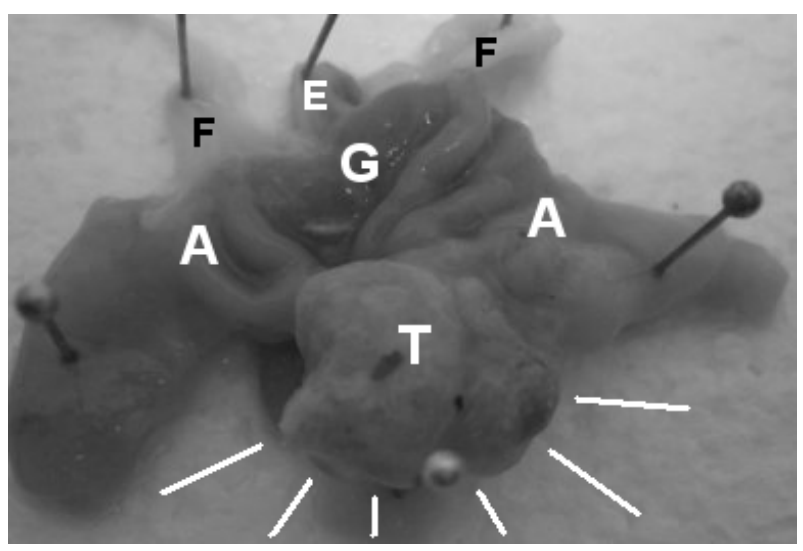

Figure 1. Macroscopic tumor. Typical macroscopic tumor found at the anastomotic area pinned at a cork board. $T$ : Tumor; A: Anastomosis; G: Glandular stomach; F: Fore stomach; E: Esophagus.

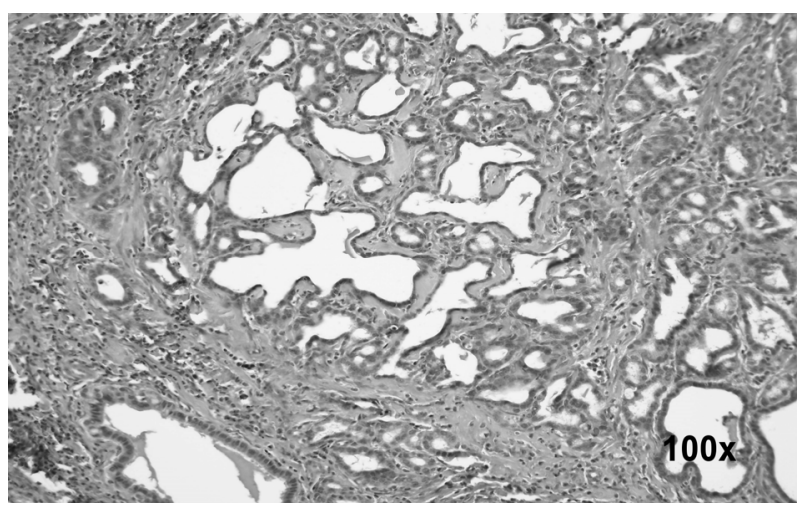

Figure 2. Adenocarcinoma at $100 \times$ magnification. Histological picture at $100 \times$ magnification of an adenocarcinoma at the gastrointestinal anastomotic area.

Table 2. Gastric lesions following 50 weeks of duodno-gastric reflux.

\begin{tabular}{ccccc}
\hline Treatment group (n) & Ulceration & ACP & Cystic dilatation & Adenocarcinoma \\
\hline Low-fat (LF) (38) & $3(7.9 \%)$ & $4(10.5 \%)$ & $5(13.2 \%)$ & $8(21 \%)$ \\
High-fat (HF) (37) & $4(10.8 \%)$ & $2(5.4 \%)$ & $7(18.9 \%)$ & $13(35 \%)$ \\
High-fat + TTA (37) & $2(5.4 \%)$ & $3(8.1 \%)$ & $0(0 \%)$ & $6(16 \%)$ \\
High-fat + FO (38) & $14(36.8 \%)^{*}$ & $18(47.4 \%)^{* *}$ & $7(18.4 \%)$ & $8(21 \%)$ \\
High-fat + TTA + FO (35) & $13(37.1 \%)^{*}$ & $13(37.1 \%)^{* *}$ & $4(11.4 \%)$ & $3(8.6 \%)^{*}$ \\
\hline
\end{tabular}

Distribution of different lesions among the various treatment groups. ACP: adenocystic proliferation. ${ }^{*} \mathrm{p}<0.01,{ }^{* *} \mathrm{p}<0.001$, compared to the high-fat group. 


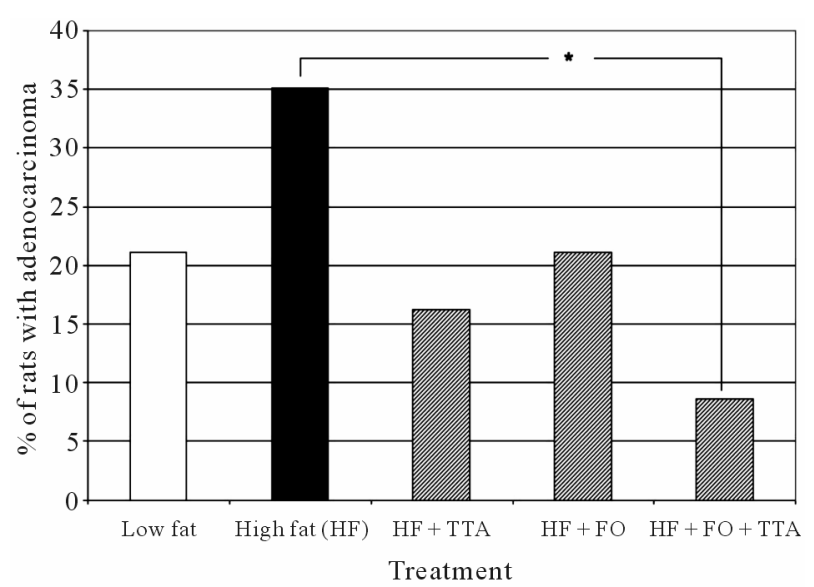

Figure 3. Distribution of adenocarcinomas. Histogram showing distribution of adenocarcinomas in the various feeding groups. ${ }^{*} \mathbf{p}<0.01$.

different bioactive fatty acids were analyzed separately (data not shown).

\subsection{Fatty Acid Profile and Anti-Inflammatory Fatty Acid Index (AIFAI) in Plasma}

The plasma fatty acid composition is shown in Table 3. TTA reduced the level of EPA (C20:5n-3) and DHA (C22:6n-3) whereas the amount of dihomo- $\gamma$-linoleic acid (DGLA, C20:3n-6) increased. The level of arachidonic acid (AA, C20:4n-6) was not affected by TTA alone, but decreased in both groups given FO (Table 3). In the FO groups, the level of AA decreased and the levels of EPA and DHA increased significantly $(\mathrm{p}<0.001)$ compared to the other treatment groups. The calculated plasma AIFAI was significantly increased in both groups receiving FO (Figure 4).

\subsection{Immunohistochemical Findings}

Immunohistochemical staining with polyclonal antibodyies against PCNA, COX-2 and p53 was performed in all cases where AC was observed (Figure 5 and Table 4). There was no significant difference between the groups in any of the three proteins (Table 4). In all groups, however, we observed signs of inflammation as evidenced by positive COX-2 staining in AC. Further, PCNA staining was positive in most AC indicating proliferation. Immunohistochemical analyses in rats without cancer revealed no differences between the various feeding groups. COX-2 always stained positive in the cytoplasm of the gastric mucosa with reduced activity in the upper middle layer of the gastric mucosa, interpreted as the area of the gut regenerative cell lineage (GRCL) [33]. PCNA stained positive in the cell nuclei in the area identified as the GRCL and the nuclei in the crypts of the intestine. p53 staining was negative. Illustration of im- munohistochemical staining in normal gastric mucosa is shown in Figure 6.

\section{Discussion}

In this experiment we tested the hypothesis if bioactive fatty acids like n-3 PUFAs found in FO and/or TTA added to a HF diet could reduce the risk of developing AC in a DGR model. We also looked at the AC development in the HF compared to the LF diet group. We found that FO as well as TTA tended to decrease the occurrence of AC. However, when combining FO with

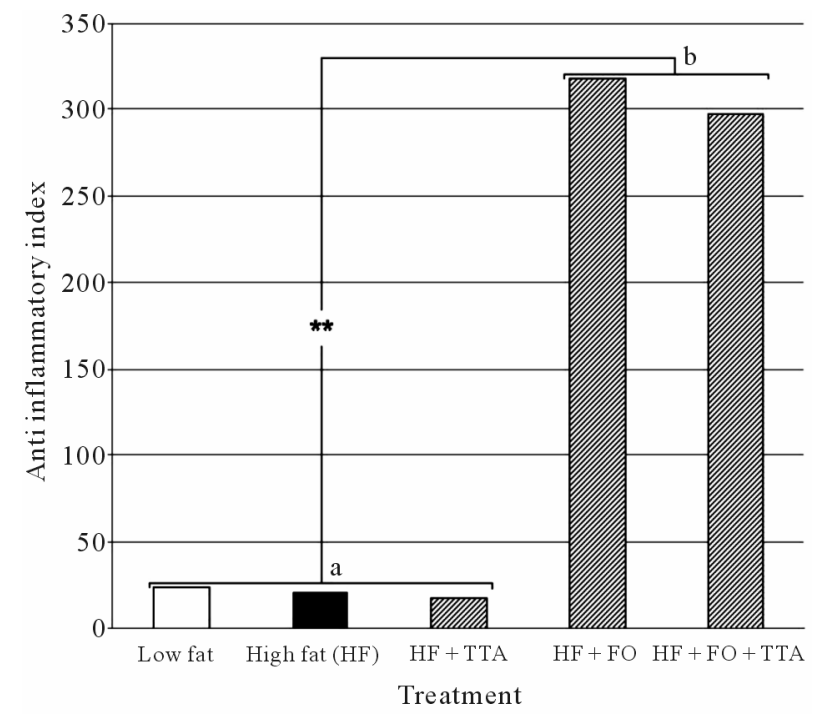

Figure 4. Anti inflammatory index in the various feeding groups. Histogram showing the anti inflammatory index for the specific treatment groups. a: treatment without FO; b: Treatment with FO. ${ }^{* *} \mathrm{p}<0.001$.

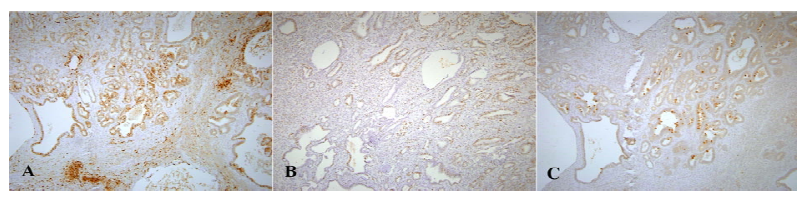

Figure 5. Immunohistochemical staining of adenocarcinomas. Immunohistochemical staining of an adenocarcinoma shown at 100× magnification. A: COX2; B: PCNA and C: p53.

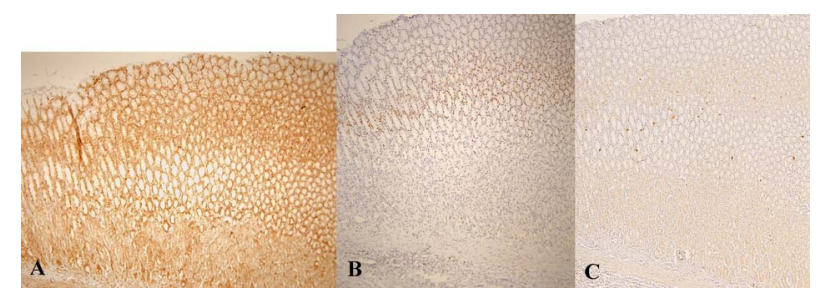

Figure 6. Immunohistochemical staining in normal gastric mucosa. Immunohistochemical staining of normal gastric mucosa tissue at $100 \times$ magnification. A: COX2; B: PCNA and $\mathrm{C}: \mathbf{p 5 3 .}$ 
Table 3. Plasma fatty acid profile.

\begin{tabular}{cccccc}
\hline \multirow{2}{*}{ Diet } & Low-fat (LF) & High-fat (HF) & HF + TTA & HF + FO & HF + FO + TTA \\
\cline { 2 - 5 } & $\mathrm{n}=38$ & $\mathrm{n}=37$ & $\mathrm{n}=37$ & $\mathrm{n}=38$ & $\mathrm{n}=35$ \\
\hline Plasma $\mu$ g/ml & & & & \\
C20:3n-6 (DGLA) & $26.3 \pm 9.1^{\mathrm{a}}$ & $19.6 \pm 6.2^{\mathrm{b}}$ & $32.0 \pm 17.0^{\mathrm{a}}$ & $7.7 \pm 2.0^{\mathrm{c}}$ & $13.5 \pm 3.8^{\mathrm{b}, \mathrm{c}}$ \\
C20:4n-6 (AA) & $613 \pm 177^{\mathrm{a}}$ & $564 \pm 140^{\mathrm{a}}$ & $495 \pm 334^{\mathrm{a}}$ & $193 \pm 37.4^{\mathrm{b}}$ & $144 \pm 37.8^{\mathrm{b}}$ \\
C20:5n-3 (EPA) & $20.6 \pm 8.3^{\mathrm{a}}$ & $11.2 \pm 4.1^{\mathrm{a}}$ & $7.9 \pm 11.3^{\mathrm{a}}$ & $365 \pm 186^{\mathrm{b}}$ & $246 \pm 102^{\mathrm{c}}$ \\
C22:5n-3 (DPA) & $16.9 \pm 6.0^{\mathrm{a}}$ & $16.9 \pm 7.3^{\mathrm{a}}$ & $4.9 \pm 2.7^{\mathrm{b}}$ & $29.5 \pm 13.9^{\mathrm{c}}$ & $15.4 \pm 6.6^{\mathrm{a}}$ \\
C22:6n-3 (DHA) & $80.1 \pm 32.1^{\mathrm{a}}$ & $70.0 \pm 23.1^{\mathrm{a}}$ & $40.8 \pm 46.4^{\mathrm{a}}$ & $220 \pm 105^{\mathrm{b}}$ & $157 \pm 67.0^{\mathrm{c}}$ \\
\hline
\end{tabular}

Plasma fatty acids are given as mean \pm SD for each treatment group with the belonging anti-inflammatory fatty acid index. AIFAI $=$ (C20:3n-6 + C20:5n-3 + C22:5n-3 + C22:6n-3) × 100/C20:4n-6. Values within a line without a common superscript are signicifantly different, $\mathrm{p}<0.05$ or higher.

Table 4. Immunohistochemical analysis of adenocarcinomas.

\begin{tabular}{cccc}
\hline Treatment group (n) & $\begin{array}{c}\text { COX-2 } \\
\text { Pos (\%) }\end{array}$ & $\begin{array}{c}\text { PCNA } \\
\text { Pos (\%) }\end{array}$ & $\begin{array}{c}\text { p53 } \\
\text { Pos (\%) }\end{array}$ \\
\hline Low-fat (8) & $6(75 \%)$ & $7(88 \%)$ & $0(0 \%)$ \\
High-fat (13) & $12(92 \%)$ & $12(92 \%)$ & $3(23 \%)$ \\
High-fat + TTA (6) & $5(83 \%)$ & $4(67 \%)$ & $0(0 \%)$ \\
High-fat + FO (8) & $5(63 \%)$ & $6(75 \%)$ & $2(25 \%)$ \\
High-fat + TTA + FO (3) & $3(100 \%)$ & $3(100 \%)$ & $1(33 \%)$ \\
\hline
\end{tabular}

The number in parenthesis by the treatment groups gives the number of AC found in the actual group. No significant differences were found between the groups.

TTA, we observed a significantly lower development of ACs compared to the HF control group ( $<<0.01)$. Our results thus demonstrate an additive effect of these bioactive lipids. When comparing the LF with the HF control group, we found a lower but not significant reduction in AC development in the LF group.

It is known that establishing a chronic gastric reflux of duodenal content in a rat model can induce gastric cancer without use of carcinogens [34]. Development of chronic inflammation is correlated to the amount of reflux. By increasing the reflux, the incidence of gastritis and cancer development increases $[34,35]$. The induction of adenocarcinomas follows the sequence of chronic inflammation, leading to metaplasia, dysplasia and finally cancer development. In our model we induced a moderate reflux and therefore we rarely found gastritis in other areas of the stomach than adjacent to the anastomosis.

There were significantly more adenocystic proliferations (ACP) in the HF + FO group and in the HF + FO + TTA treatment group (Table 2). Miwa et al. [35] has suggested that ACPs are precancerous lesions. According to these findings it might be considered whether TTA treatment alone is able to lower the frequency of ACP. FO seems to reduce the risk of ACP transforming into AC but this treatment did not fully prevent its occurrence. The underlying mechanisms of the anti-tumor activity of FO are not fully understood. There is some evidence indicating that its n-3 PUFAs, EPA and DHA, can reduce inflammation and promote apoptosis in cancer cells [20$22,36]$. One of these mechanisms is believed to be that the n-6 PUFA, AA (20:4n-6) is the main substrate for eicosanoids like prostaglandins, leucotriens and thromboxans. These eicosanoids are mediators and regulators of inflammation [37,38]. The AA metabolism and its metabolites are known targets for anti-inflammatory drug therapy. This includes non-steroidal anti-inflammatory drugs (NSAIDs) that inhibit the COX-2 activity. This effect was documented in a similar reflux model where gastritis and cancer development was reduced signifycantly by treatment with meloxicam, a specific COX-2inhibitor [13]. However, there was no effect of TTA and FO on the protein level of COX-2, as the COX-2 staining was equal in all treatment groups in specimens without AC lesions. An inhibition of the COX-2 activity instead of the protein level could not be excluded though. The same observation was seen on staining against PCNA and p53, where there was no difference between the groups.

It has been shown that supplementation of FO, which is rich in EPA and DHA, induce a time-dependent incorporation of these $n-3$ PUFAs into the cell membrane phospholipids $[37,39,40]$. Once present in the membranes, EPA and DHA will then dislodge AA available for synthesis of AA-derived eicosanoids. EPA and DHA are also able to act as a substrate for the cyclooxygenase and the 5-lipoxygenase [37]. These eicosanoids produced from EPA and DHA have a slightly modified structure and exert less inflammatory activity than AA-derived eicosanoids. EPA can also reduce proliferation by directly inducting apoptosis as demonstrated in cell culture models [41,42].

The plasma fatty acid profile in each treatment group (Table 3) showed a significantly increase of the AIFAI 
(Figure 4) with a highly significant difference $(\mathrm{p}<0.001)$ between the groups receiving FO compared to the groups without FO supplemented. This observation supports the hypothesis that EPA and DHA are able to exert anti-inflammatory effects in this model.

The rationale behind the combination of FO and TTA was that they theoretically might have additive antiproliferative effects, even if it has been reported that TTA reduce the EPA and DHA levels in plasma [43]. In the present study, the content of EPA and DHA in plasma increased after FO supplementation, whereas the content of dihomo- $\gamma$-linoleic acid (DGLA, C20:3n-6) was reduced. The synthesis of eicosanoids depends on the availability of the 20-carbon PUFAs, either arriving via circulation or arising from local tumor production catalyzed by delta 5 and delta 6 desaturases. FO reduced the content of AA in plasma, possibly making AA less available for eicosanoid synthesis, which could modulate inflammation [39]. After FO supplementation, the AIFAI increased in both groups (Figure 4). As shown in Figure 3, TTA reduced the incidence of AC even more than FO alone. As the AIFAI was unchanged after TTA treatment, we therefore hypothesize that the anti-cancer effect of TTA is different than for FO, and that is may be due to induction of apoptosis, possibly mediated by mitochondrial alterations and changes in inflammation signalling as outlined in the background section. Since no signifycant difference was found with the ANOVA test when treatments with different bioactive fatty acids were done separately, we assume that the pathways are different and that there is an additive effect of TTA and FO to reduce the occurrence of AC.

\section{Conclusion}

In conclusion, a diet containing both FO and TTA has an additive and reducing effect on the development of AC in a DGR rat model. The exact mechanism is not clear and further studies are needed to elucidate this.

\section{Acknowledgements}

The authors gratefully acknowledge Anne Aarsand, Inger Vikøyr, Christ Berge and Laila Vaardal for laboratory assistance and Åshild Åsebø for taking care of all the animals. This project was supported financially by Western Norway Regional Health Authority and the Nordic Centre of Excellence-MitoHealth.

\section{REFERENCES}

[1] J. Ferlay, P. Autier, M. Boniol, M. Heanue, M. Colombet and P. Boyle, "Estimates of the Cancer Incidence and Mortality in Europe in 2006," Annals of Oncology, Vol. 18, No. 3, 2007, pp. 581-592. doi:10.1093/annonc/mdl498
[2] D. M. Parkin, F. Bray, J. Ferlay and P. Pisani, "Global Cancer Statistics, 2002,” Cancer Journal for Clinicians, Vol. 55, No. 2, 2005, pp. 74-108. doi:10.3322/canjclin.55.2.74

[3] C. Prinz, S. Schwendy and P. Voland, "H Pylori and Gastric Cancer: Shifting the Global Burden,” World Journal of Gastroenterology, Vol. 12, No. 34, 2006, pp. 54585464.

[4] H. Brenner, D. Rothenbacher and V. Arndt, "Epidemiology of Stomach Cancer,” Methods in Molecular Biology, Vol. 472, 2009, pp. 467-477. doi:10.1007/978-1-60327-492-0_23

[5] K. D. Crew and A. I. Neugut, "Epidemiology of Gastric Cancer," World Journal of Gastroenterology, Vol. 12, No. 3, 2006, pp. 354-362.

[6] S. Bahmanyarand and W. Ye, "Dietary Patterns and Risk of Squamous-Cell Carcinoma and Adenocarcinoma of the Esophagus and Adenocarcinoma of the Gastric Cardia: a Population-Based Case-control Study in Sweden,” Nutrition and Cancer, Vol. 54, No. 2, 2006, pp. 171-178. doi:10.1207/s15327914nc5402_3

[7] C. A. Gonzalez, P. Jakszyn, G. Pera, A. Agudo, S. Bingham and J. R. Quiros, "Meat Intake and Risk of Stomach and Esophageal Adenocarcinoma within the European Prospective Investigation into Cancer and Nutrition (EPIC)," Journal of the National Cancer Institute, Vol. 98, No. 5, 2006, pp. 345-354. doi:10.1093/jnci/djj071

[8] S. T. Mayne, H. A. Risch, R. Dubrow, W. H. Chow, M. D. Gammon and J. F. Fraumeni Jr., "Nutrient Intake and Risk of Subtypes of Esophageal and Gastric Cancer," Cancer Epidemiology, Biomarkers \& Prevention, Vol. 10, No. 10, 2001, pp. 1055-1062.

[9] S. A. Navarro Silvera, S. T. Mayne, H. Risch, M. D. Gammon, T. L. Vaughan and W. H. Chow, "Food Group Intake and Risk of Subtypes of Esophageal and Gastric Cancer," International Journal of Cancer, Vol. 123, No. 4, 2008, pp. 852-860. doi:10.1002/ijc.23544

[10] P. A. van den Brandt, A. A. Botterweck and R. A. Goldbohm, "Salt Intake, Cured Meat Consumption, Refrigerator Use and Stomach Cancer Incidence: A Prospective Cohort Study (Netherlands)," Cancer Causes Control, Vol. 14, No. 5, 2003, pp. 427-438. doi:10.1023/A:1024979314124

[11] E. Riboli and T. Norat, "Cancer Prevention and Diet: Opportunities in Europe,” Public Health Nutrition, Vol. 4, No. 2B, 2001, pp. 475-484. doi:10.1079/PHN2001158

[12] C. Liu and R. M. Russell, "Nutrition and Gastric Cancer Risk: An Update,” Nutrition Reviews, Vol. 66, No. 5, 2008, pp. 237-249. doi:10.1111/j.1753-4887.2008.00029.x

[13] M. Oba, K. Miwa, T. Fujimura, S. Harada, S. Sasaki and T. Hattori, "Chemoprevention of Glandular Stomach Carcinogenesis through Duodenogastric Reflux in Rats by a COX-2 Inhibitor,” International Journal of Cancer, Vol. 123, No. 7, 2008, pp. 1491-1498. doi:10.1002/ijc.23742

[14] M. Oba, K. Miwa, T. Fujimura, S. Harada, S. Sasaki, K. 
Oyama and T. Hattori, “A Selective Cyclooxygenase-2 Inhibitor Prevents Inflammation-related Squamous Cell Carcinogenesis of the Forestomach via Duodenogastric Reflux in Rats," Cancer, Vol. 115, No. 2, 2009, pp. 454464. doi:10.1002/cncr.23990

[15] H. Chen, M. H. Ward, B. I. Graubard, E. F. Heineman, R. M. Markin and K. L. Tucker, "Dietary Patterns and Adenocarcinoma of the Esophagus and Distal Stomach," American Journal of Clinical Nutrition, Vol. 75, No. 1, 2002, pp. 137-144.

[16] F. L. Haven, "The Effect of Cod-Liver Oil on Tumor Growth,” American Journal of Cancer, Vol. 27, 1936, pp. 95-98.

[17] V. Maillard, P. Bougnoux, P. Ferrari, M. L. Jourdan, M. Pinault and V. Chajes, "N-3 and N-6 Fatty Acids in Breast Adipose Tissue and Relative Risk of Breast Cancer in a Case-control Study in Tours, France,” International Journal of Cancer, Vol. 98, No. 1, 2002, pp. 78-83. doi:10.1002/ijc.10130

[18] P. Terry, P. Lichtenstein, M. Feychting, A. Ahlbom and A. Wolk, "Fatty Fish Consumption and Risk of Prostate Cancer," Lancet, Vol. 357, No. 9270, 2001, pp. 17211812. doi:10.1016/S0140-6736(00)04889-3

[19] P. Terry, A. Wolk, H. Vainio and E. Weiderpass, "Fatty Fish Consumption Lowers the Risk of Endometrial Cancer: a Nationwide Case-control Study in Sweden," Cancer Epidemiology, Biomarkers \& Prevention, Vol. 11, No. 1, 2002, pp. 143-145.

[20] J. Cheng, K. Ogawa, K. Kuriki, Y. Yokoyama, T. Kamiya and S. Tokudome, "Increased Intake of n-3 Polyunsaturated Fatty Acids Elevates the Level of Apoptosis in the Normal Sigmoid Colon of Patients Polypectomized for Adenomas/Tumors," Cancer Letters, Vol. 193, No. 1, 2003, pp. 17-24. doi:10.1016/S0304383502007176

[21] E. D. Courtney, S. Matthews, C. Finlayson, D. Di Pierro, A. Belluzzi and R. J. Leicester, "Eicosapentaenoic Acid (EPA) Reduces Crypt Cell Proliferation and Increases Apoptosis in Normal Colonic Mucosa in Subjects with a History of Colorectal Adenomas," International Journal of Colorectal Disease, Vol. 22, No. 7, 2007, pp. 765-776. doi:10.1007/s00384-006-0240-4

[22] Y. Y. Fan, Y. Zhan, H. M. Aukema, L. A. Davidson, L. Zhou and R. S. Chapkin, "Proapoptotic Effects of Dietary (n-3) Fatty Acids Are Enhanced in Colonocytes of Manganese-Dependent Superoxide Dismutase Knockout Mice,” Journal of Nutrition, Vol. 139, No. 7, 2009, pp. 13281332. doi:10.3945/jn.109.106203

[23] K. Berge, K. J. Tronstad, P. Bohov, L. Madsen and R. K. Berge, "Impact of Mitochondrial Beta-Oxidation in Fatty Acid-mediated Inhibition of Glioma Cell Proliferation,” Journal of Lipid Research, Vol. 44, No. 1, 2003, pp. 118-127. doi:10.1194/jlr.M200312-JLR200

[24] K. Berge, K. J. Tronstad, E. N. Flindt, T. H. Rasmussen, L. Madsen, K. Kristiansen and R. K. Berge, "Tetradecylthioacetic Acid Inhibits Growth of Rat Glioma Cells ex vivo and in vivo via PPAR-dependent and PPAR-independent Pathways,” Carcinogenesis, Vol. 22, No. 11, 2001, pp. 1747-1755. doi:10.1093/carcin/22.11.1747

[25] K. J. Tronstad, O. Bruserud, K. Berge and R. K. Berge,
“Antiproliferative Effects of a Non-Beta-Oxidizable Fatty Acid, Tetradecylthioacetic Acid, in Native Human Acute Myelogenous Leukemia Blast Cultures,” Leukemia, Vol. 16, No. 11, 2002, pp. 2292-2301. doi:10.1038/sj.leu.2402698

[26] K. J. Tronstad, B. T. Gjertsen, C. Krakstad, K. Berge, O. T. Brustugun and R. K. Berge, "Mitochondrial-Targeted Fatty Acid Analog Induces Apoptosis with Selective Loss of Mitochondrial Glutathione in Promyelocytic Leukemia Cells," Chemistry \& Biology, Vol. 10, No. 7, 2003, pp. 609-618. doi:10.1016/S1074-5521(03)00142-X

[27] P. O. Iversen, D. R. Sorensen, K. J. Tronstad, O. A. Gudbrandsen, A. C. Rustan and C. A. Drevon, "A Bioactively Modified Fatty Acid Improves Survival and Impairs Metastasis in Preclinical Models of Acute Leukemia," Clinical Cancer Research, Vol. 12, No. 11, 2006, pp. 35253531. doi:10.1158/1078-0432.CCR-05-2802

[28] M. Suo, K. Mukaisho, A. Shimomura, H. Sugiha and T. Hattori, "Thioproline Prevents Carcinogenesis in the Remnant Stomach Induced by Duodenal Reflux," Cancer Letters, Vol. 237, No. 2, 2006, pp. 256-262. doi:10.1016/j.canlet.2005.06.019

[29] E. G. Bligh and W. J. Dyer, "A Rapid Method of Total Lipid Extraction and Purification," Canadian Journal of Biochemistry and Physiology, Vol. 37, No. 8, 1959, pp. 911-917. doi:10.1139/059-099

[30] M. Kates, “General Analytical Procedures. In Techniques in Lipidology," M. Dates Elsevier Science Publishers, Amsterdam, 1986, pp. 112-185.

[31] S. R. Chavali, W. W. Zhong, T. Utsunomiya and R. A. Forse, "Decreased Production of Interleukin-1-Beta, Prostaglandin-E2 and Thromboxane-B2, and Elevated Levels of Interleukin-6 and -10 Are Associated with Increased Survival during Endotoxic Shock in Mice Consuming Diets Enriched with Sesame Seed Oil Supplemented with Quil-A saponin,” International Archives of Allergy and Immunology, Vol. 114, No. 2, 1997, pp. 153-160. doi:10.1159/000237661

[32] T. Utsunomiya, S. R. Chavali, W. W. Zhong and R. A. Forse, "Effects of Sesamin-Supplemented Dietary Fat Emulsions on the ex Vivo Production of Lipopolysaccharide-Induced Prostanoids and Tumor Necrosis Factor Alpha in Rats," American Journal of Clinical Nutrition, Vol. 72, No. 3, 2000, pp. 804-808.

[33] K. Mukaisho, K. Miwa, H. Kumagai, M. Bamba, H. Sugihara and T. Hattori, "Gastric Carcinogenesis by Duodenal Reflux through Gut Regenerative Cell Lineage,” Digestive Diseases and Sciences, Vol. 48, No. 11, 2003, pp. 2153-2158. doi:10.1023/B:DDAS.0000004519.26201.a4

[34] K. Miwa, T. Hattori and I. Miyazaki, "Duodenogastric Reflux and Foregut Carcinogenesis,” Cancer, Vol. 75, No. 6, 1995, pp. 1426-1432. doi:10.1002/1097-0142(19950315)75:6+<1426::AID-CN CR2820751506>3.0.CO;2-\#

[35] K. Miwa, H. Hasegawa, T. Fujimura, H. Matsumoto, R. Miyata and T. Hattori, "Duodenal Reflux through the Pylorus Induces Gastric Adenocarcinoma in the Rat," Carcinogenesis, Vol. 13, No. 12, 1992, pp. 2313-2316. doi:10.1093/carcin/13.12.2313 
[36] S. P. Mehta, A. P. Boddy, J. Cook, V. Sams, E. K. Lund and M. Rhodes, "Effect of n-3 Polyunsaturated Fatty Acids on Barrett's Epithelium in the Human Lower Esophagus," American Journal of Clinical Nutrition, Vol. 87, No. 4, 2008, pp. 949-956.

[37] W. Fetterman Jr. and M. M. Zdanowicz, "Therapeutic Potential of n-3 Polyunsaturated Fatty Acids in Disease," American Journal of Health-System Pharmacy, Vol. 66, No. 13, 2009, pp. 1169-1179. doi:10.2146/ajhp080411

[38] S. L. Tilley, T. M. Coffman and B. H. Koller, "Mixed Messages: Modulation of Inflammation and Immune Responses by Prostaglandins and Thromboxanes," Journal of Clinical Investigation, Vol. 108, No. 1, 2001, pp. 1523.

[39] M. J. James, R. A. Gibson and L. G. Cleland, "Dietary Polyunsaturated Fatty Acids and Inflammatory Mediator production," American Journal of Clinical Nutrition, Vol. 71, No. 1, 2000, pp. 343S-348S.

[40] R. I. Sperling, A. I. Benincaso, C. T. Knoell, J. K. Larkin,
K. F. Austen and D. R. Robinson, "Dietary Omega-3 Polyunsaturated Fatty Acids Inhibit Phosphoinositide Formation and Chemotaxis in Neutrophils," Journal of Clinical Investigation, Vol. 91, No. 2, 1993, pp. 651-660. doi:10.1172/JCI116245

[41] H. Heimli, C. Giske, S. Naderi, C. A. Drevon and K. Hollung, "Eicosapentaenoic Acid Promotes Apoptosis in Ramos Cells via Activation of Caspase-3 and -9," Lipids, Vol. 37, No. 8, 2002, pp. 797-802. doi:10.1007/s11745-002-0963-6

[42] H. Heimli, K. Hollung, and C. A. Drevon, "Eicosapentaenoic Acid-Induced Apoptosis Depends on Acyl CoASynthetase,” Lipids, Vol. 38, No. 3, 2003, pp. 263-268. doi:10.1007/s11745-003-1059-z

[43] D. K. Asiedu, L. Froyland, H. Vaagenes, O. Lie, A. Demoz and R. K. Berge, "Long-Term Effect of Tetradecylthioacetic Acid: A Study on Plasma Lipid Profile and Fatty Acid Composition and Oxidation in Different Rat Organs,” Biochimica et Biophysica Acta, Vol. 1300, No. 2, 1996, pp. 86-96. 